\title{
Vitamin D fortification and seasonality of birth in type 1 diabetic cases: D-tect study - ERRATUM
}

\author{
R. Jacobsen, M. Moldovan, A. A. Vaag, E. Hypponen and B. L. Heitmann \\ doi:10.1017/S2040174415007849, published by Cambridge University Press, 27 October 2015.
}

First published online 18 April 2016

We earlier reported that there was no seasonality of birth among Danish boys with type 1 diabetes (T1D), born before the cancellation of margarine vitamin $\mathrm{D}$ fortification programme in Denmark in 1985, opposed to the seasonality of birth found among boys with T1D born after the programme cancellation. As boys born before the fortification cancelation were exposed prenatally to extra vitamin $\mathrm{D}$ coming from fortified margarine, whereas boys born after the cancelation were not, we concluded that the vitamin $\mathrm{D}$ in fortified foods was sufficient to overcome the effects of natural seasonal variations in vitamin $\mathrm{D}$ levels, and in such a way affect the later T1D risk. ${ }^{1}$ We later found that the higher maternal exposure to sunshine during pregnancy was associated with the lower risk of T1D in the same sample of Danish boys. ${ }^{2}$ We then re-ran the seasonality of birth models with additional adjustment for pregnancy sunshine hours, as well as compared these hours between the boys born before (i.e. exposed) and after (i.e. unexposed) the cancelation of vitamin $\mathrm{D}$ fortification programme. We found that after the adjustment for pregnancy sunshine hours, the seasonality of birth in T1D cases was now absent in both groups. Indeed, when comparing boys born in spring vs. boys born in autumn in the exposed group (i.e. born during a two-year-period before the fortification cancellation) the Hazard ratio (HR; 95\% CI) for developing T1D before age 15 years was 0.78 (0.39-1.59). Similarly, HR; 95\% CI in the unexposed group (i.e. born during a two-year-period after the fortification cancelation) was $0.93(0.49-1.77)$. For boys who developed T1D at age 5-9 years, the respective results were: $2.10(0.56-7.87)$ for the exposed and $2.80(0.81-9.60)$ for unexposed. We also found that there were more sunshine hours during the pregnancy of the mothers to boys exposed to fortification compared to the mothers of unexposed boys: mean difference (SD) being 38 (0.8) hours. These findings suggest that pregnancy sunshine was an omitted relevant factor in assessing the association between the prenatal exposure to vitamin $\mathrm{D}$ fortification and the seasonality of birth patterns. While vitamin $\mathrm{D}$ fortification may still have some relevance in the analysis, most probably it was the larger amount of pregnancy sunshine hours among mothers giving birth before the fortification cancelation in 1985, compared to mothers giving birth afterwards that primary stood behind the reduction of T1D risk in boys born in spring compared to those born in autumn, i.e. seasonality of birth observed. ${ }^{1}$ Sunshine may have had its effect on T1D risk by both vitamin D-related and vitamin D-unrelated (e.g. via infections or lifestyle) pathways. $^{2}$

\section{References}

1. Jacobsen R, Moldovan M, Vaag AA, Hypponen E, Heitmann BL. Vitamin $\mathrm{D}$ fortification and seasonality of birth in type 1 diabetic cases: D-tect study. J Dev Orig Health Dis. 2016; 7, 114-119.

2. Jacobsen R, Frederiksen P, Heitmann BL. Exposure to sunshine early in life prevented development of type 1 diabetes in Danish boys. J Pediatr Endocrinol Metab. 2015 [Epub ahead of print]. 